\title{
Pigeon hypersensitivity pneumonitis: immunohistochemical demonstration of the causative antigen in the lung
}

\section{Diana Elodia Aguilar León ${ }^{a}$, Virginia Novelo Retana ${ }^{b}$, Rogelio Hernández-Pandoa, Juana Arellanoc, Liliana García Ortizc, Fernando Cano Valled, *Erasmo Martínez-Corderoe, The Interdisciplinary group for the study of extrinsic allergic alveolitis}

\footnotetext{
${ }^{a}$ Department of Experimental Pathology, National Institute of Medical Sciences and Nutrition, Mexico City, Mexico

${ }^{b}$ Head, Clinical Service of Respiratory Diseases, General Hospital of Mexico

Fellow in Biomedical Research, National Institute of Respiratory Diseases, Mexico City, Mexico

${ }^{d}$ General Director, National Institute of Respiratory Diseases, Mexico

e Head, Laboratory of Research in Autoimmunity, National Institute of Respiratory Diseases, Mexico
}

Received 3rd August 2006; accepted 26th March 2007

\begin{abstract}
A number of clinicopathological manifestations may define the presence of hypersensitivity pneumonitis. Histological study is used to establish the diagnosis and to differentiate the disease from other respiratory disorders. This case report suggests that immunohistological demonstration of the causative antigen in the lung may be a useful diagnostic approach in cases of pigeon hypersensitivity pneumonitis. A 52 year-old woman was studied. She had a prior history of pigeon exposure, and lived in an area with a high prevalence of tuberculosis. Her clinical presentation, respiratory function tests and imaging studies revealed a predominantly interstitial lung disease. The results of antiavian antibodies, bronchoalveolar analysis, and other laboratory parameters were non-diagnostic. A lung biopsy showed a prominent granulomatous reaction with a sarcoid-like appearance in some areas, and an interstitial infiltration constituted by lymphocytes, plasma cells and foamy macrophages. Although the disease manifestations were compatible with hypersensitivity pneumonitis, we decided to study the causal antigen by immunohistochemistry. The use of a polyclonal antibody raised against pigeon serum showed a predominant cytoplasmic immunostaining in multinucleated giant cells and histiocytes from lung granulomas. Other respiratory disorders were reasonably excluded. Previous exposure to a known antigen may support the diagnosis of hypersensitivity pneumonitis. Although the inhalation of organic dusts may be clinically evident, the aetiology is commonly evaluated by different challenge tests or immunological methods. We propose that the study of pigeon antigen by immunohistochemistry may be used as part of the diagnostic approach for hypersensitivity pneumonitis.

(C) 2007 General Practice Airways Group. All rights reserved.

DE Aguilar León, et al. Prim Care Resp J 2007; 16(4): 252-256.

doi:10.3132/pcrj.2007.00046
\end{abstract}

Keyw ords extrinsic allergic alveolitis, pigeon breeder's disease, bird fancier's lung, granulomatous interstitial pneumonia, multinucleated giant cells.

\section{Introduction}

Pigeon hypersensitivity pneumonitis (HP) is an immunologically-mediated disease caused by organic antigens of avian origin. ${ }^{1-3}$ Since no pathognomonic finding defines the diagnosis, establishing the diagnosis depends on a combination of clinical, laboratory, lung function and imaging abnormalities, as well as several histopathological features. ${ }^{1.4} \mathrm{~A}$ variety of characteristic manifestations have been used to define the illness: the presence of restrictive ventilatory impairment; bronchoalveolar lavage (BAL) lymphocytosis; typical opacities shown on high resolution computerised tomography (HRCT) scanning; and a prominent immunological response to the causative antigen. ${ }^{3-6}$

The relation of HP to organic dusts derived mainly from feathers and/or avian droppings has been clearly documented, ${ }^{2}$ and it is not surprising that a number of

\footnotetext{
* Corresponding author: Research Unit, National Institute of Respiratory Diseases, Calzada de Tlalpan 4502, Mexico, City, 14080, Mexico. Tel: +52(55)56664539 Fax: +52(55)56654623 Email: emartinezcordero@yahoo.com.mx; emartinezcordero@hotmail.com
} 
diagnostic procedures have involved the demonstration of such environmental exposure. Provocation tests with inhaled pigeon serum, and the study of antibodies or different $\mathrm{T}$ cell abnormalities associated with bird antigens have been widely used for the diagnosis and proposed as predictive criteria of HP by some groups. ${ }^{7-10}$ The use of lung biopsy has also been invaluable to corroborate the presence of $\mathrm{HP}$, and different reports have recognised the characteristic appearance of mononuclear bronchiolitis, diffuse interstitial infiltrates of lymphocytes and/or plasma cells, scattered small nonnecrotising granulomas, aggregates of epitheliod histiocytes and/or multinucleated giant cells, and in some instances centriacinar and perilobular fibrosis. ${ }^{11-14,15}$ Nevertheless, a number of difficulties may arise during the differentiation of the disease using an exclusively histopathologic approach. ${ }^{14,10}$

A heterogeneous array of respiratory entities may be similar to HP in terms of their morphologic features, and several studies have pointed out that the differential diagnosis include the broad group of idiopathic interstitial pneumonias, a diversity of granulomatous conditions, some drug hypersensitivity pneumonitis conditions, and a variety of occupational, infectious, and neoplastic lung disorders. ${ }^{13-19}$

This case report concerns a patient who presented with a respiratory illness suggestive of HP. Since there was no clear evidence that the disease was the result of pigeon exposure and since lung biopsy revealed a prominent granulomatous reaction which suggested other diagnostic considerations, an immunohistochemical technique was used in order to identify the causative antigen and to define the pathological diagnosis. The report indicates that the presence of pigeon antigen may be histologically demonstrated in HP.

\section{Case report}

A 52 year-old woman was admitted to our Institute in November 2005 with a four-year history of progressive dyspnoea on exertion and cough occasionally accompanied by mucoid sputum, together with symptoms of general malaise and loss of appetite. Four years previously, she had initially been treated by her primary care physician with amoxicillin for three weeks because of a presumed infection but there was no significant clinical improvement. During the next two-and-a-half years her illness followed an insidious course with slowly progressive clinical deterioration and no acute exacerbations. In the winter of 2004, a private pneumonologist had suggested the possibility of miliary tuberculosis (TB) particularly since the patient lived in an area with a high TB prevalence and since she had an abnormal chest radiograph. Antituberculous antibiotics including rifampicin, isoniazid and pyrazinamide had been prescribed, but after five weeks her treatment was arbitrarily stopped and no information about further diagnostic studies was available.
Clinical deterioration continued over the next seven months, until her dyspnoea became severe enough to cause difficulties in climbing five to six flights of stairs. On admission, a persistent and predominantly dry cough together with mild chest discomfort was noted. There was a history of intermittent exposure to pigeons for at least seven years. The family and occupational background was unremarkable, and there was no other antecedent history of note including smoking habit, alcohol intake, drug addictions, and inhalation of additional organic or inorganic dusts. Her past medical history did not reveal allergic conditions, infectious disorders, pharmacologic reactions, or respiratory abnormalities associated with avian contact.

On examination the patient looked chronically ill. She was dyspnoeic with a respiratory rate of 26 breaths/min, and her heart rate was 88 beats/min. Inspiratory crackles were heard over both lung fields, and except for mild clubbing of the fingers we did not find any other physical abnormalities. Arterial blood gases in air showed normal $\mathrm{PO}_{2}, \mathrm{PCO}_{2}$, and $\mathrm{pH}$ values, and an oxygen saturation of $91 \%$ which decreased to $85 \%$ after walking. Laboratory tests showed a raised erythrocyte sedimentation rate $(39 \mathrm{~mm} / \mathrm{hr})$, mild eosinophilia (9\%; normal $3-6 \%)$, and a platelet count of $490 \times 10^{\%} / 1$ (normal 150-450 $\times 10 \%$ ). Other findings included hypergammaglobulinemia (1.9 g/dl; normal 0.5-1.5 g/dl), high lgG (3610 mg/dl; normal 850-1868 mg/dl) and IgA levels (585 mg/dl; normal 80.7-549 mg/dl), elevation of C-reactive protein $(4.1 \mathrm{mg} / \mathrm{dl}$; normal $0.3-1.7 \mathrm{mg} / \mathrm{dl})$, and positive rheumatoid factor (1:80, latex agglutination). Liver enzymes and serum electrolytes were normal. Serologic tests for infectious agents that may produce lung disease, including common respiratory viruses, and all routine blood and urine cultures, yielded negative results. The level of angiotensin converting enzyme was normal, and a purified protein derivative skin test was positive. No precipitating antibodies were found using thermophylic actinomycetes as well as pigeon serum and droppings preparations as target antigens. ${ }^{20}$ Although the values of antiavian IgG antibodies by ELISA exceeded the reference range of healthy controls with no bird exposure, the comparison to asymptomatic breeders showed overlapping levels. ${ }^{20,21}$ Unfortunately, study of pigeon sensitisation through the antigen-induced lymphocyte proliferation test was not available.

Pulmonary function tests revealed a predominantly restrictive pattern with a low forced vital capacity (FVC) of 1.64 litres (43\% of predicted), a forced expiratory volume in one second $\left(\mathrm{FEV}_{1}\right)$ of 1.42 litres (52\% of predicted), an $\mathrm{FEV}_{1} / \mathrm{FVC}$ ratio of 0.87 , and a reduced diffusion capacity for carbon monoxide ( $\mathrm{DLCO}, 12 \mathrm{ml} / \mathrm{min} / \mathrm{mm} \mathrm{Hg}, 54 \%$ of predicted). Diffuse and bilateral reticulonodular infiltrates were detected on chest radiograph, and the HRCT showed 
centrilobular micronodules diffusely distributed in both lung fields. Studies of BAL fluid obtained by bronchoscopy revealed a cellularity of $1.2 \times 10^{9}$ leucocytes/l with $72 \%$ macrophages, $24 \%$ lymphocytes, and $4 \%$ eosinophils. The CD4/CD8 $T$ cells ratio was 1.2. Additionally, no organisms were seen on acid fast bacilli or fungal stains, and the microbiological cultures produced negative results. However, this evaluation did not include a transbronchial biopsy.

The histopathological analysis of surgical lung biopsy specimens revealed numerous and well organised nonnecrotising granulomas. Although there was a plentiful formation of multinucleated giant cells and epitheliod histiocytes diffusely distributed, a sarcoid-like appearance was also evident in some areas in which the cellular reaction occurred alongside bronchovascular bundles (see Figure 1). The interstitial infiltration was prominent and constituted by lymphocytes, plasma cells and foamy macrophages. There was a moderate fibrosis in some areas of the lung, and no changes consistent with necrosis, vasculitis or viral cytopathic effects were found. The polarised light microscopy excluded the possibility of birefringent material, and the routine stains, including those for acid fast bacilli, fungi and amyloid, were all negative. Additionally, the study of $M$. tuberculosis and $M$. avium by in situ $P C R^{22}$ did not reveal abnormalities. Although the diagnosis of HP was reasonably demonstrated through the traditional diagnostic approach, the serological tests used to identify an aetiological cause were not conclusive. Therefore, the presence of avian antigen was histopathologically studied through an immunoperoxidase system; it revealed strong cytoplasmic immunostaining of multinucleated giant cells and histiocytes from lung granulomas. Some interstitial activated and foamy macrophages also showed cytoplasmic immunostaining (see Figure 1).

The histopatological and immunohistochemical features were compatible with a diagnosis of pigeon HP. The patient was initially treated with prednisone $40 \mathrm{mg} / \mathrm{day}$, and was discharged home with the advice to avoid pigeon exposure. A gradual clinical and functional respiratory improvement occurred after two months of corticosteroid administration and elimination of avian contact. Unfortunately, the patient was lost to follow-up in March 2006, and we were unable to obtain further information about the disease evolution.

Immunohistochemistry: The rabbit polyclonal antibody (patent pending) that led us to detect the presence of the causative antigen in the lung of HP with a biotinylated goat anti-rabbit IgG, and a commercial immunohistochemical staining method (Vectastain ABC system, Vector, USA) was raised against high and low molecular weight proteins of pigeon serum and purified by affinity chromatography (Affigel 10 Pharmacia, USA). Since a variety of pigeon
Figure 1. Representative histologic and immunohistochemistry findings of the lung biopsy.

(A) Well formed non-necrotic granulomas constituted by lymphocytes, macrophages and numerous

multinucleated giant cells. There is also extensive interstitial inflammatory infiltrate predominantly formed by lymphocytes and macrophages (H/E x100). (B) Immunohistochemical demonstration of pigeon antigen. Multinucleated giant cells and macrophages show strong cytoplasmic immunostaining (x200). (C) Control immunohistochemistry section without primary antibody incubation (x200).

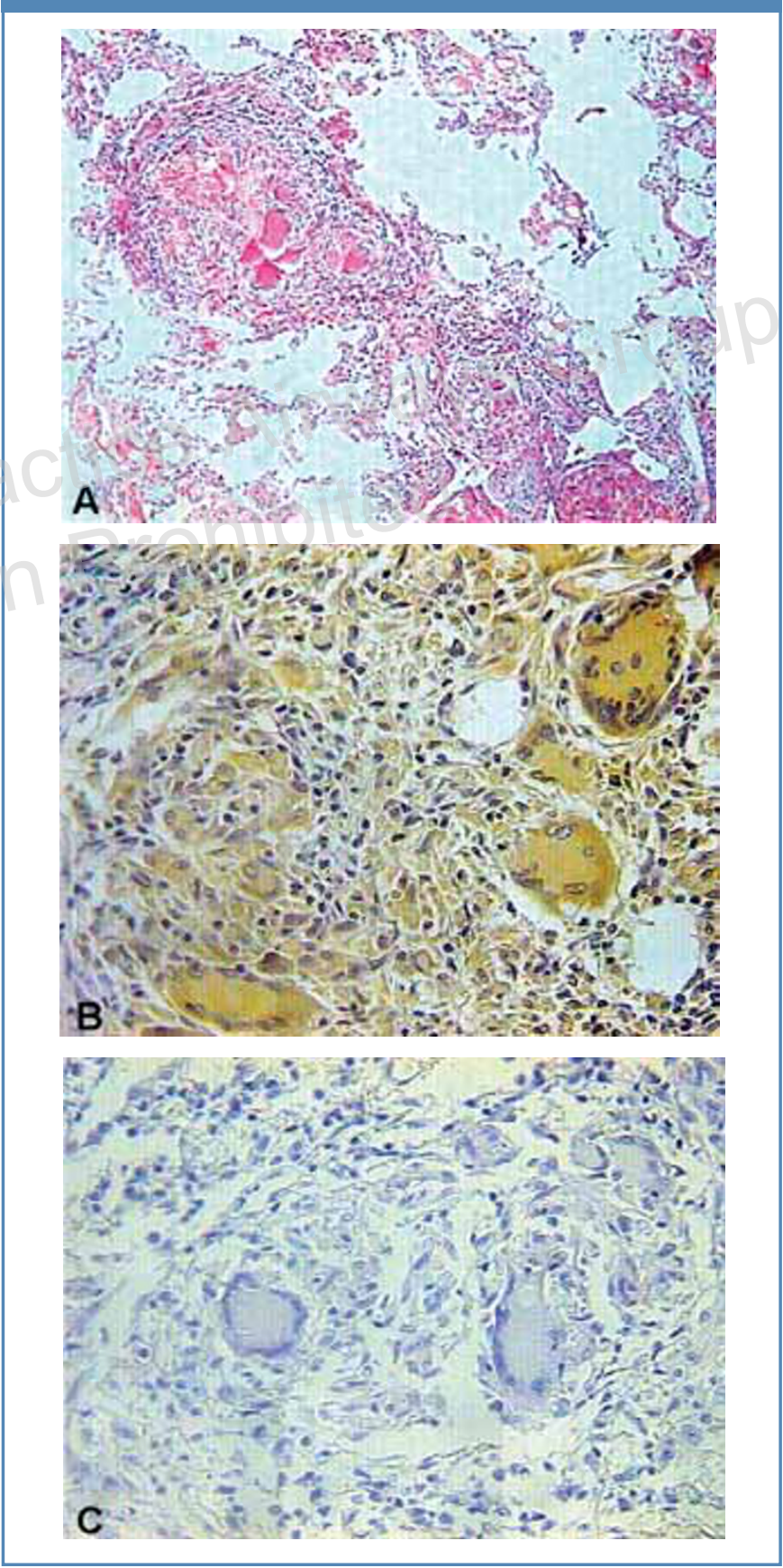


antigens have been implicated in the development of the disease, we used an antibody that recognised a full range of avian components through Western blot $^{20}$ using sera and droppings of pigeon origin as target antigens. This immunoglobulin did not reveal cross-reaction with normal goat serum that was included as a blocking agent in the immunoperoxidase system, and with a pool of human sera obtained from healthy donors. In addition, the immunohistochemical study for avian antigen revealed negative results using lung biopsy specimens of respiratory abnormalities different from HP, including sarcoidosis, usual interstitial pneumonitis, tuberculosis, and lung fibrosis secondary to scleroderma.

\section{Discussion}

A number of HP manifestations are useful in achieving a diagnosis but no unique feature seems to be pathognomonic. The presence of some clinical, lung function, or imaging findings, and BAL abnormalities that have been considered typical of HP, may be found in other respiratory entities. No unifying criteria have supported the diagnostic value of challenge tests with avian products; a variable immunological response according to the clinical presentation and the course of HP, depending on the source of pigeon antigen, has been described. $4,7,23-26$.

This histological study is no exception. Although the use of lung biopsy has been helpful in defining the presence of HP in cases with no clear evidence of avian exposure, and can corroborate the diagnosis of the insidious and recurrent subsets of chronic disease or evaluate patients who may fulfil the HP criteria but showed no improvement to antigen avoidance and/or therapy, it has been shown that a variety of diagnostic difficulties may arise during the pathological evaluation. ${ }^{1,13-17,26}$

In our patient, the clinical history, laboratory findings, pulmonary function tests and HRCT, together with the histological analysis, immunohistochemistry and in situ PCR, not only supported the diagnosis of HP but reasonably excluded the presence of HP secondary to exposure to thermophylic actinomycetes and $\mathrm{M}$. avium. ${ }^{22} \mathrm{~A}$ heterogeneous group of lung disorders has been found to be histologically similar to HP; the morphologic spectrum may change according to the stage of the illness, non-specific interstitial findings have been reported, and a variable pathological expression appears to distinguish different HP subsets. ${ }^{14,18.27-29}$

Since the histopathological abnormalities in HP are not pathognomonic, and the appearance of some atypical or unusual features can lead to different diagnostic considerations, study of the causative antigen in the lung may be useful in order to differentiate the disease, particularly in patients with morphological findings similar to other respiratory conditions such as non-specific interstitial involvement, a marked bronchial infiltrate, extensive fibrosis, or a plentiful formation of multinucleated giant cells, ${ }^{13-19}$ as occurred in this case. In this case, several diseases which may show a prominent granulomatous interstitial pneumonia like drug reactions, fungal and atypical mycobacterial infections, pneumoconiosis, or vasculitis and Sjögren's syndrome ${ }^{19}$ - were excluded.

The possibility of demonstrating a definitive cause in HP is invaluable in terms of the diagnostic approach, in particular when the clinical, functional, imaging or other distinctive manifestations of the illness are missing. Different groups have recognised the association between avian exposure and the development of the disease: ${ }^{8-10}$ the clinical history may provide evidence of previous inhalation of pigeon materials and its relationship with an immediate onset of the illness; the environmental examination of avian dusts has confirmed the presence of subacute HP in some patients; appropriate challenge tests with the causative antigen may support the diagnosis of chronic disease; and the sensitisation to avian products has been documented through different immunological methods during the study of acute, subacute, or chronic HP. ${ }^{4,724-26}$ In this context, immunohistochemical evaluation may be a useful alternative for establishing the definitive cause of the disease during the diagnostic work-up of a patient with suspected pigeon HP.

The demonstration of pigeon antigen in the lung of HP induced by this extrinsic organic exposure is not entirely surprising; other causative antigens may be detected during the histopathologic appraisal. Different thermophilic microorganisms have been identified in farmer's HP through immunohistochemistry or by microbiological tests, and several specialised methods such as scanning electron microscopy, specific culture assays, energy-dispersive spectroscopy, and restriction fragment-length polymorphism (RFLP) have confirmed causal agents using lung biopsy specimens from hypersensitivity disorders secondary to the inhalation of a variety of organic and inorganic antigens. ${ }^{13,30-35}$ We are now studying whether the presence of pigeon antigen in the lung may have further diagnostic value, given that the disease has shown a broad histopathological spectrum.

\section{Acknow ledgements}

This study was supported by a grant from the National Council of Science and Technology, Mexico.

\section{Conflict of interest declaration}

There are no conflicts of interest to declare.

\section{References}

1. Kline JN, Hunninghake GW, Hypersensitivity pneumonitis and pulmonary infiltrates with eosinophilia. In Braunwald E, Fauci AS (eds): Harrison's 
Principles of Internal Medicine (ed 15). New York, NY, McGraw-Hill, In, 2001. pp 1463-67.

2. McSharry C, Anderson K, Boyd G. A review of antigen diversity causing lung disease among pigeon breeders. Clin Exp Allergy 2000;30:1221-9.

3. Patel AM, Ryu JH, Reed CE. Hypersensitivity pneumonitis: current concepts and future questions. J Allergy Clin Immunol 2001;108:661-70.

4. Mohr LC. Hypersensitivity pneumonitis. Curr Opin Pulm Med 2004;10:401-04.

5. Glazer CS, Rose CS, Lynch DA. Clinical and radiologic manifestations of hypersensitivity pneumonitis. J Thorac Imaging 2002;17:261-72.

6. Lacasse $Y$, Selman M, Costabel U, et al. Clinical diagnosis of hypersensitivity pneumonitis. Am J Respir Crit Care Med 2003;168:952-8.

7. Ohtani $Y$, Kojima K, Sumi $Y$, Sawada M, et al. Inhalation provocation tests in chronic bird fancier's lung. Chest 2000;118:1382-9.

8. Schuyler M, Cormier Y. The diagnosis of hypersensitivity pneumonitis. Chest 1997;64:307-09.

9. American Thoracic Society/European Respiratory Society. International Multidisciplinary Consensus Classification of the Idiopathic Interstitial Pneumonias. Am J Respir Crit Care Med 2002;165:227-304

10. Dangman $\mathrm{KH}$, Cole SR, Hodgson MJ, et al. The hypersensitivity pneumonitis diagnostic index: use of non-invasive testing to diagnose hypersensitivity pneumonitis in metalworkers. Am J Ind Med 2002;42:150-62.

11. Richerson HB, Bernstein IL, Fink JN, et al. Guidelines for the clinical evaluation of hypersensitivity pneumonitis. J Allergy Clin Immunol 1989;84:839-44.

12. Coleman A, Colby TV. Histologic diagnosis of extrinsic allergic alveolitis. Am J Surg Pathol 1988;12:514-18

13. Colby TV. Surgical pathology of non-neoplastic lung disease. Mod Pathol 2000; 13:343-58

14. Travis WD, Matsui K, Moss J, et al. Idiopathic nonspecific interstitial pneumonia: prognostic significance of cellular and fibrosing patterns: survival comparison with usual interstitial pneumonia and desquamative interstitial pneumonia. Am J Surg Pathol 2000;24:19-33.

15. Ohtani Y, Saiki S, Kitaichi M, et al. Chronic bird fancier's lung: histopathological and clinical correlation. An application of the 2002 ATS/ERS consensus classification of the idiopathic interstitial pneumonias. Thorax 2005;60:665-71.

16. Nicholson AG. Pulmonary lymphoproliferative disorders. Curr Diag Pathol 2000;6:130-9.

17. Nicholson AG. Classification of idiopathic interstitial pneumonias: making sense of the alphabet soup. Histopathology 2002;41:381-91.

18. Yousem SA, Dacic S. Idiopathic bronchiolocentric interstitial pneumonia. Mod Pathol 2002;15:1148-53.

19. Cheung OY, Muhm JR, Helmers RA, et al. Surgical pathology of granulomatous interstitial pneumonia. Ann Diagn Pathol 2003;7:127-38.
20. Martinez-Cordero E, Aguilar Leon DE, Retana VN. IgM antiavian antibodies in sera from patients with pigeon breeder's disease. J Clin Lab Anal 2000;14:20107.

21. Aguilar Leon DE, Novelo Retana V, Martinez-Cordero E. Anti-avian antibodies and rheumatoid factor in pigeon hypersensitivity pneumonitis. Clin Exp Allergy 2003;33:226-32.

22. Hernández-Pando R, Jeyanathan M, Mengistu G, et al. Persistence of DNA from Mycobacterium tuberculosis in superficially normal lung tissue during latent infection. Lancet 2000;356:2133-8.

23. Krasnick J, Meuwissin HJ, Nakao MA, et al. Hypersensitivity pneumonitis: problems in diagnosis. J Allergy Clin Immunol 1996;97:1027-30.

24. Matar LD, McAdams PH, Sporn TA. Hypersensitivity pneumonitis. AJR 2000;174:1061-6.

25. Morell F, Roger A, Cruz MJ. Usefulness of specific skin tests in the diagnosis of hypersensitivity pneumonitis. J Allergy Clin Immunol 2002;1106:939.

26. Zacharisen MC. Idiopathic interstitial pneumonia: are we missing hypersensitivity pneumonitis? Ann Allergy Asthma Immunol 2002;88:4-6.

27. Cottin V, Donsbeck A, Revel D, et al. Nonspecific interstitial pneumonia: individualization of a clinicopathological entity in a series of 12 patients. Am Rev Respir Crit Care Med 1998;158:1286-93.

28. Suda T, Chida K, Hayakawa H, et al. Development of bronchus-associated lymphoid tissue in chronic hypersensitivity pneumonitis. Chest 1999;115:35763.

29. Vourlekis JS, Schwarz MI, Cool CD, et al. Nonspecific interstitial pneumonitis as the sole histologic expression of hypersensitivity pneumonitis. Am J M ed 2002; 112:490-3.

30. Wenzel FJ, Emanuel DA, Lawton BR, et al. Isolation of the causative agent of farmer's lung. Ann Allergy 1964;22:533-40.

31. Wenzel FJ. Emanuel DA, Lawton BR. Pneumonitis due to micromonospora vulgaris (farmer's lung). Am Rev Resp Dis 1967;95:652-5.

32. Reijula K, Sutinen S. Immunohistochemical identification of aspergillus fumigatus in farmer's lung. Acta Histochem 1984;75:211-13.

33. Reijula K, Sutinen S. Detection of antigens in lung biopsies by immunoperoxidase staining in extrinsic allergic bronchioalveolitis (EABA). Acta Histochem 1985;76:121-5.

34. Embil J, Warren P, Yakrus $M$, et al. Pulmonary illness associated with exposure to Mycobacterium-avium complex in hot tub water. Hypersensitivity pneumonitis or infection? Chest 1997;111:813-16.

35. Safirstein BH, Klukowicz A, Miller R, et al. Granulomatous pneumonitis following exposure to the World Trade Center collapse. Chest 2003;123:30104.

\section{Available online at $\mathbf{h t t p}: / / \mathbf{w} \mathbf{w} \mathbf{w}$.thepcrj.org}

